Original Paper

\title{
Effects of Misalignment of High Speed Flexible Coupling on the Fighter Aircraft Transmission Characteristics
}

\author{
Nagesh Samikkanu and Abu Muhammed Junaid Basha \\ Combat Vehicles Research and Development Establishment, \\ Avadi, Chennai 600 054. India \\ nge0207@gmail.com
}

\begin{abstract}
The Fighter aircraft transmission system consists of a light weight, High Speed Flexible Coupling (HSFC) known as Power Take-Off shaft (PTO) for connecting Engine gearbox (EGB) with Accessory Gear Box (AGB). The HSFC transmits the power through series of specially contoured metallic annular thin flexible plates whose planes are normal to the torque axis. The HSFC operates at high speed ranging from 10,000 to $18,000 \mathrm{rpm}$. The HSFC is also catered for accommodating larger lateral and axial misalignment resulting from differential thermal expansion of the aircraft engine and mounting arrangement. The contoured titanium alloy flexible plates are designed with a thin cross sectional profile to accommodate axial and parallel misalignment by the elastic material flexure. This paper investigates the effect of misalignment on the transmission characteristics of the HSFC couplings. A mathematical model for the HSFC coupling with misalignment has been developed for analyzing the torque transmission and force interaction characteristics. An extensive testing has been conducted for validating characteristics of the designed coupling under various misalignment conditions. With this the suitability of the model adapted for the design iteration of HSFC development is validated. This method will reduce the design iteration cycles of HSFC and can be extended for the similar development of flexible couplings.
\end{abstract}

Keywords: Flexible Couplings, Misalignment, rotor dynamics, system dynamics and simulation, Experimental validation, Failure Prevention.

\section{Introduction}

The Fighter aircraft transmission system consists of a light weight, High Speed Flexible Coupling (HSFC) known as power takeoff shaft for connecting Engine gearbox (EGB) with Accessory Gear Box (AGB). The HSFC transmits the power through series of specially contoured metallic annular thin flexible plates whose planes are normal to the torque axis as shown in Fig 1. The HSFC operates at high speed ranging from 10,000 to $18,000 \mathrm{rpm}$ from the EGB to AGB. The HSFC also catered for accommodating larger angular and axial misalignment resulting from differential thermal expansion of the aircraft engine and mounting arrangement. The contoured titanium alloy flexible plates are designed with a thin cross sectional profile to accommodate axial and parallel misalignment by the elastic material flexure. The parallel misalignment and axial displacement distorts or bends the flexible plates which are designed with thinner cross section to maintain lower bending and axial stresses for a given torque capacity. Due to misalignment, the HSFC is subjected to high cyclic stresses. The present work investigates the effect of misalignment of the HSFC on the Fighter Aircraft Transmission Characteristics. The mathematical model for the HSFC coupling with misalignment is constructed for the purpose of deriving the equations governing the torque transmission and force interaction characteristics. The test apparatus is constructed for verifying the static and dynamic characteristics of the designed coupling under various misalignment conditions. Good agreement between numerical simulation and experimental results is obtained are presented.

\subsection{Configuration of HSFC}

The schematic view of the High speed flexible coupling is depicted in Figure 1. The flexible plate cross sectional profile may be straight, contoured, tapered or convoluted. The flexible plate used for HSFC designed to suit the operating conditions, misalignment requirements, enveloping dimensional restrictions and safety [1]. The size of the coupling is based on the mean and maximum operation torques. The imposed axial, parallel and rotational misalignments resulting in enhanced stress level on the 
flexible plates. These are investigated and confirmed by extensive static and dynamic stress analysis .Two flexible plate packs assembled in the input and output end of HSFC connected by a centre tube to share the loads equally during operating condition.

\subsection{HSFC Misalignment}

The HSFC misalignment occurs when the centerlines of rotation of two power transmitting shafts are not in line with each other. The misalignment is the deviation of relative shaft position from a collinear axis of rotation, measured at the point of rotation [2]. The misalignment may result in excessive vibrations [3]. In the aircraft transmission system, as the engine and accessories drives are mounted on different bases, the dynamic operating conditions results in parallel misalignment of HSFC. The axial misalignment of the HSFC is due to the thermal expansion of engine during operation. The HSFC transmits torque through a stack of thin metal flexible plates. The free span of the flexible plates deflects to accommodate angular misalignment and allow minor axial shaft movement. The HSFC misalignment [3] occurs when the centerlines of rotation of two power transmitting shafts are not in line with each other. The misalignment is the deviation of relative shaft position from a collinear axis of rotation (measured at the points of rotation). The coupling misalignment conditions are shown in Table 1.

Table 1 Coupling Misalignment

\begin{tabular}{c|l|c}
\hline \hline S1. No & \multicolumn{1}{|c}{ Misalignment } & Misalignment Condition \\
\hline 1 & $\begin{array}{l}\text { In parallel misalignment the two axes are parallel but at a } \\
\text { relatively small distance apart. }\end{array}$ & $\begin{array}{l}\text { In axial misalignment shafts are positioned at the same } \\
\text { axis but separated by a small distance. }\end{array}$ \\
\hline
\end{tabular}

In the aircraft transmission system as the engine drives and accessories drive are mounted on different bases, the dynamic operating conditions result in parallel misalignment of HSFC. The axial misalignment of the HSFC is due to the thermal expansion of engine during operation.

\section{Influence of Articulation length on parallel Misalignment}

The configuration of HSFC is shown in Fig 1. The flexible plates pack accommodates the misalignment imposed on the system. The articulation length is the distance between the center of flexible plate packs attached at either end of the center tube. The Fig 2 shows articulation length of HSFC. The location of flexible plates packs influences the amount of misalignment it can accommodate.

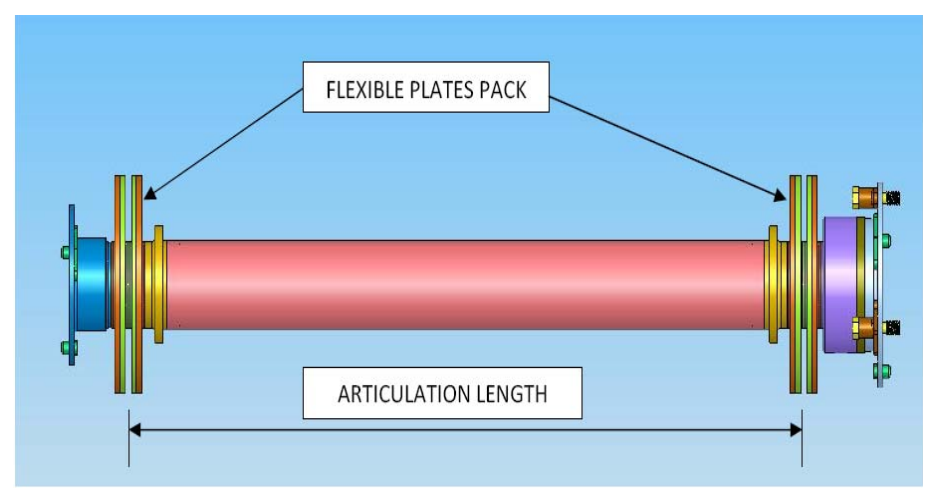

Fig. 1 Configuration of HSFC

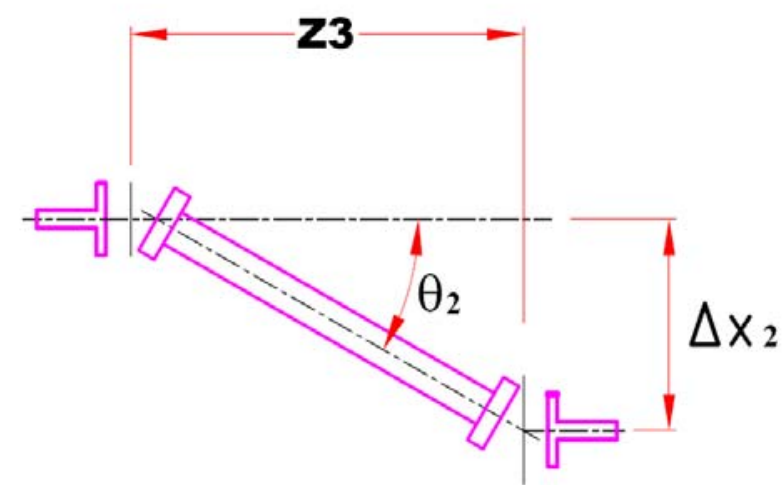

Fig. 2 Articulation length $\left(Z_{3}\right)$ of HSFC

The relation between the off- set distance and articulation length is given by the eq (1).

$$
\Delta X_{2}=\tan \left(\theta_{2}\right) \cdot Z_{8}
$$

In case of axial misalignment the articulation length has no influence on the amount of misalignment. But in case of Parallel misalignment the articulation length determines the magnitude of parallel misalignment distance. The table 2 shows the rotating angle and offset distance with various articulation lengths. 
Table 2 Influence of articulation length of HSFC

\begin{tabular}{r|r|r|r|r}
\hline \hline \multirow{2}{*}{$\begin{array}{l}\text { Rotating } \\
\text { angle }\left(\theta_{2}\right)\end{array}$} & \multicolumn{4}{|c}{ Articulation length, $\mathrm{Z}_{3}(\mathrm{~mm})$} \\
\cline { 2 - 5 } & \multicolumn{4}{|c}{ Resulting Off set distance $(\mathrm{mm})$} \\
\cline { 2 - 5 } 0.5 & 3.052739 & 3.488845 & 3.706897 & 3.92495 \\
\hline 1.0 & 6.105246 & 6.977424 & 7.413513 & 7.849602 \\
\hline 1.5 & 9.157288 & 10.46547 & 11.11956 & 11.77366 \\
\hline 2.0 & 12.20863 & 13.95272 & 14.82477 & 15.69682 \\
\hline 2.5 & 15.25905 & 17.43892 & 18.52885 & 19.61878 \\
\hline 3.0 & 18.30831 & 20.92378 & 22.23152 & 23.53925 \\
\hline 3.5 & 21.35617 & 24.40705 & 25.93249 & 27.45793 \\
\hline 4.0 & 24.40241 & 27.88847 & 29.6315 & 31.37453 \\
\hline 4.5 & 27.44679 & 31.36776 & 33.32825 & 35.28873 \\
\hline 5.0 & 30.48908 & 34.84467 & 37.02246 & 39.20025 \\
\hline
\end{tabular}

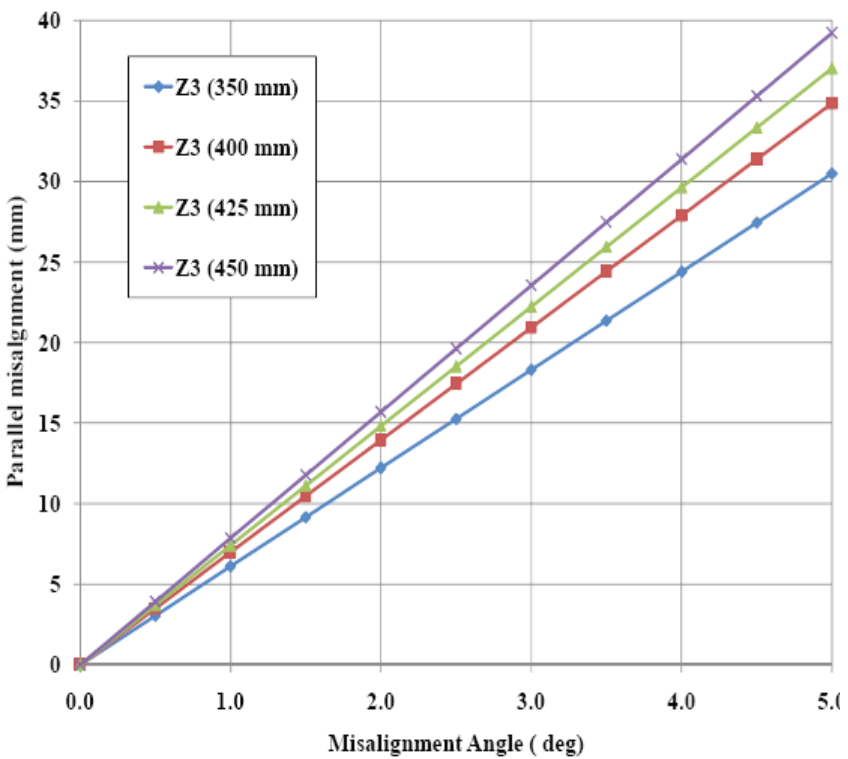

Fig. 3 Influence of articulation length

The typical case with rotating angel of $1.5^{\circ}$ the increase in resulting off-set distance for the articulation length of $450 \mathrm{~mm}$ is $22.2 \%$ more when compared to $350 \mathrm{~mm}$ of articulation length. So, for the same rotating angle $\left(\theta_{2}\right)$, increase in articulation length $\left(Z_{3}\right)$ resulting in increase in resulting off-set distance which is to be accommodated by the HSFC. With the lower rotating angle $\left(\theta_{2}\right)$, the corresponding bending stress induced on the flexible elements will be less thereby increase in the HSFC service life can be realized.

\section{Coupling Misalignment}

The reaction forces and moments developed due to parallel Misalignment has been analysed by Gibbons [4]. The rotor bearing system has been modelled and the reaction forces, moments developed due to flexible coupling misalignment are derived by A. S Sekhar and B. S Prabhu [5]. With these, in the present study, the reaction forces, moments developed due to HSFC misalignment is arrived. The coordinates of parallel misalignment of HSFC is shown in Fig 4. During parallel misalignment the centre-lines $Z_{1}$ and $Z_{2}$ are misaligned. The torque is applied at input end and that the rotation is in the same direction as the applied torque. The reaction forces and moments which the HSFC exerts on the end supports are as given below.

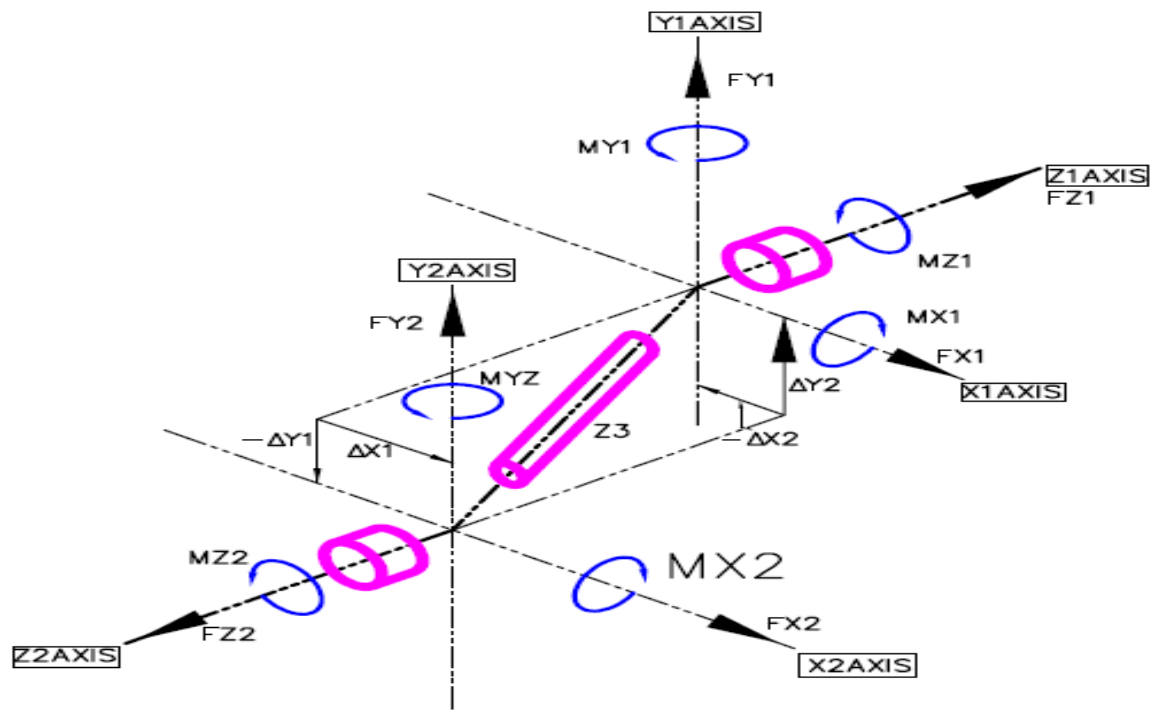

Fig. 4 Coordinates of parallel misalignment of HSFC

The centre-line of the center tube, a coupling spacer is connecting the HSFC centerlines, with intersection points on the coupling articulation center. The values and the directions of displacements, $\Delta \mathrm{X} 2$ and $\Delta \mathrm{Y} 2$ can be sub suited to simulate the misalignment .Assuming that $\mathrm{Z1}$ is the axis of driving end and the torque is applied as shown in Fig 4 where the rotation is in the same direction as the applied torque, the reaction forces and moments that the coupling exerts on the end supports are arrived from the system equation of motion of HSFC. 


\subsection{Equation of motion of HSFC}

The following are reaction forces \& moments that are developed on account of parallel misalignment

$$
\begin{aligned}
& \mathrm{MX}_{1}=\mathrm{T}_{q} \sin \theta_{1}+\mathrm{K}_{b} \Phi_{1} \\
& \mathrm{MY}_{1}=\mathrm{T}_{q} \sin \Phi_{1}-\mathrm{K}_{b} \quad \theta_{1} \\
& \mathrm{MX}_{2}=\mathrm{T}_{q} \sin \theta_{2}-\mathrm{K}_{b} \Phi_{2} \\
& \mathrm{MY}_{2}=\mathrm{T}_{q} \sin \Phi_{2}+\mathrm{K}_{b} \quad \theta_{2} \\
& F X_{1}=\frac{-M Y_{0}-N Y_{n}}{Z_{3}} \\
& \mathrm{FY}_{1}=\frac{-N Z_{\mathrm{g}}+N V_{\mathrm{N}}}{z_{\mathrm{s}}} \\
& \mathrm{FZ}_{1}=\mathrm{Ka} \Delta \mathrm{Z}+\mathrm{Ka}(\Delta \mathrm{Z})^{3} \\
& \Theta_{1}=\sin ^{-1}\left(\frac{\Delta X_{1}}{x_{0}}\right) \\
& \theta_{2}=\sin ^{-1}\left(\frac{\Delta X_{2}}{Z_{R_{i}}}\right) \\
& \Phi_{1}=\sin ^{-1}\left(\frac{\Delta y_{1}}{z_{8}}\right) \\
& \Phi_{2}=\sin ^{-1}\left(\frac{\Delta \gamma_{1}}{Z_{g}}\right)
\end{aligned}
$$

Considering the linear bending and axial spring rates for the HSFC are assumed. Therefore,

$$
\begin{aligned}
& \mathrm{FX}_{2}=-\mathrm{FX}_{1} \\
& \mathrm{FY}_{2}=-\mathrm{FY}_{1} \\
& \mathrm{FE}_{2}=\mathrm{FE}_{1}
\end{aligned}
$$

From these forces in each direction can be obtained .For the design iteration of HSFC, initial guess value can be subsuited for comparative calculations. Thus the reaction forces and moments can be calculated by considering system equation of motion.

\subsection{Bending stiffness Vs Forces and moments}

In this the articulation length and induced torques are kept constant and the bending stiffness of the HSFC is varied to study the resulting forces and moments. The bending stiffness also influences the bending natural frequency of the HSFC [6]. The results are shown in Table 3.

Table 3 Variation in Bending stiffness Vs Forces and moments

\begin{tabular}{r|r|r|l|l|l|l|l|l|l|l}
\hline \hline $\begin{array}{l}\text { Torque } \\
(\mathrm{Tq})\end{array}$ & $\mathrm{k}_{\mathrm{b}}$ & $\mathrm{Mx}_{1}$ & $\mathrm{My}_{1}$ & $\mathrm{Mx}_{2}$ & $\mathrm{My}_{2}$ & $\mathrm{FX}_{1}$ & $\mathrm{FY}_{1}$ & $\Delta \mathrm{X}_{2}$ & $\Delta \mathrm{Y}_{2}$ & $\mathrm{Z}_{3}$ \\
\hline & & & & & & & & & & \\
100 & 0 & 0 & 0.0000 & 2.8235 & 2.8235 & -0.0066 & 0.0066 & 12 & 12 & 425 \\
\hline 100 & 950 & 0 & 0.0000 & -24.0036 & 29.651 & -0.0698 & -0.0565 & 12 & 12 & 425 \\
\hline 100 & 1900 & 0 & 0.0000 & -50.8307 & 56.478 & -0.1329 & -0.1196 & 12 & 12 & 425 \\
\hline 100 & 2850 & 0 & 0.0000 & -77.6578 & 83.305 & -0.1960 & -0.1827 & 12 & 12 & 425 \\
\hline 100 & 3325 & 0 & 0.0000 & -91.0713 & 96.718 & -0.2276 & -0.2143 & 12 & 12 & 425 \\
\hline 100 & 4750 & 0 & 0.0000 & -131.3119 & 136.96 & -0.3223 & -0.3090 & 12 & 12 & 425 \\
\hline
\end{tabular}

As the bending stiffness increases the corresponding moment and forces on the end support are increasing. The bending stiffness variation Vs moments and bending stiffness Vs forces are shown in Fig 5 \& 6. 


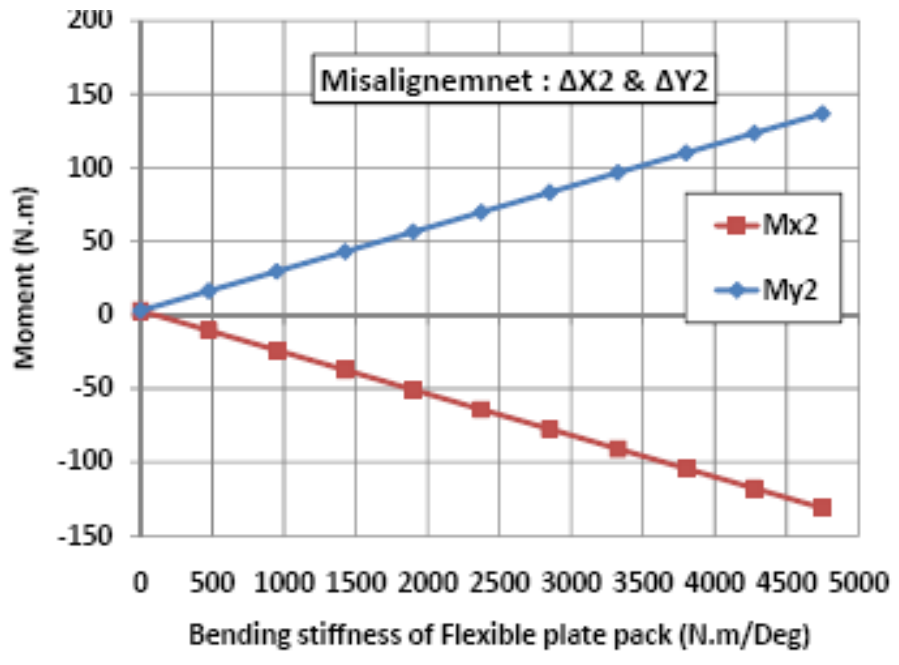

Fig 5. Bending stiffness Vs end moments

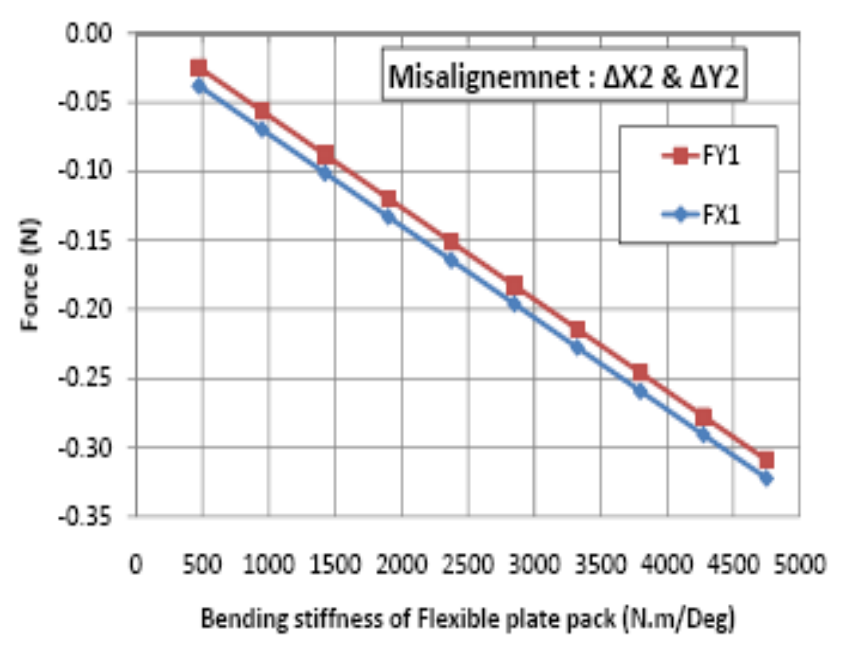

Fig 6. Bending stiffness Vs Forces

\subsection{Articulation length Vs Forces and moments}

In this the induced Torque and bending stiffness is kept constant and articulation length of the HSFC is varied. The resulting forces and moments are calculated for the typical HSFC are given in Table 4. The articulation length Vs end moments and articulation length Vs reaction forces are shown in Fig $7 \& 8$.

Table 4 Articulation length Vs Forces and end moments

\begin{tabular}{c|l|l|l|l|l|l|l|l}
\hline \hline Torque $(\mathrm{Tq})$ & $\mathrm{K}_{\mathrm{b}}$ & $\mathrm{Mx}_{2}$ & $\mathrm{My}_{2}$ & $\mathrm{FX}_{1}$ & $\mathrm{FY}_{1}$ & $\Delta \mathrm{X}_{2}$ & $\Delta \mathrm{Y}_{2}$ & $\mathrm{Z}_{3}$ \\
\hline 100 & $4.75 \mathrm{E}+03$ & -186.0507 & 194.051 & -0.64684 & -0.6202 & 12 & 12 & 300 \\
\hline 100 & $4.75 \mathrm{E}+03$ & -171.7322 & 179.117 & -0.55113 & -0.5284 & 12 & 12 & 325 \\
\hline 100 & $4.75 \mathrm{E}+03$ & -159.4605 & 166.318 & -0.47519 & -0.4556 & 12 & 12 & 350 \\
\hline 100 & $4.75 \mathrm{E}+03$ & -148.8260 & 155.226 & -0.41394 & -0.3969 & 12 & 12 & 375 \\
\hline 100 & $4.75 \mathrm{E}+03$ & -139.5214 & 145.521 & -0.36380 & -0.3488 & 12 & 12 & 400 \\
\hline 100 & $4.75 \mathrm{E}+03$ & -131.3119 & 136.959 & -0.32226 & -0.3090 & 12 & 12 & 425 \\
\hline 100 & $4.75 \mathrm{E}+03$ & -124.0150 & 129.348 & -0.28744 & -0.2756 & 12 & 12 & 450 \\
\hline 100 & $4.75 \mathrm{E}+03$ & -117.4865 & 122.539 & -0.25798 & -0.2473 & 12 & 12 & 475 \\
\hline
\end{tabular}

As the articulation length increases the corresponding moment and forces on the end support are decreasing. It will be advantageous to have more articulation length to reduce the end moments and forces as shown in the articulation length Vs end moments and articulation length Vs forces as shown in Fig $7 \& 8$.

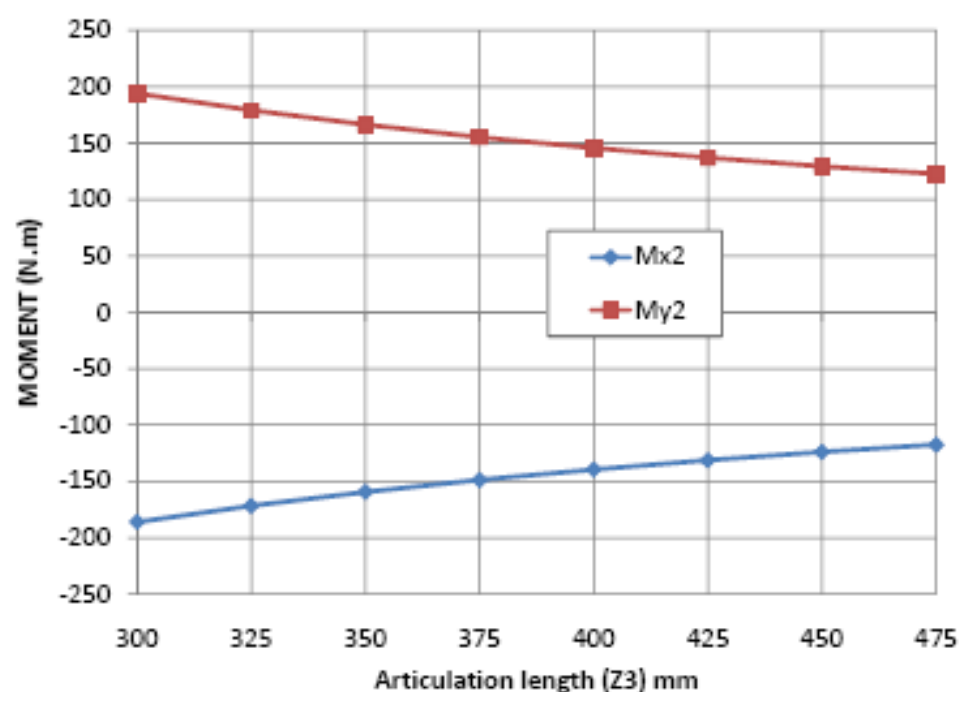

Fig 7. Articulation length Vs end moments

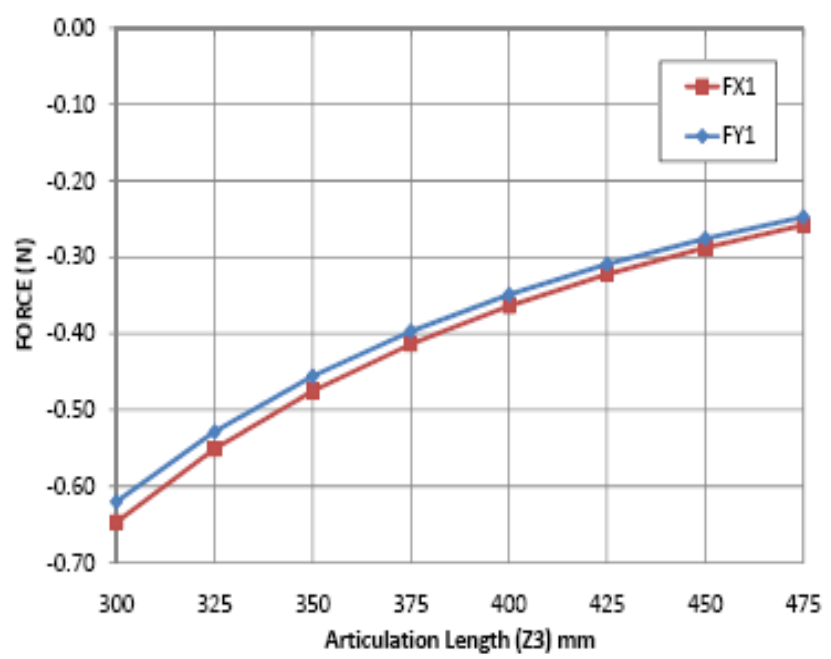

Fig 8. Articulation length Vs Forces 


\subsection{Parallel misalignment Vs Forces and moments}

In this the induced Torque and bending stiffness and Articulation length are kept constant and with variation in Parallel misalignment of the HSFC, the resulting forces and moments are calculated for the typical HSFC is given in Table 5 .

Table 5 Parallel misalignment Vs Forces and moments

\begin{tabular}{c|l|c|c|c|c|c|c|c|c}
\hline \hline $\begin{array}{c}\text { Torque } \\
(\mathrm{Tq})\end{array}$ & $\mathrm{k}_{\mathrm{b}}$ & $\mathrm{Mx}_{1}$ & $\mathrm{My}_{1}$ & $\mathrm{Mx}_{2}$ & $\mathrm{My}_{2}$ & $\mathrm{FX}_{1}$ & $\mathrm{FY}_{1}$ & $\Delta \mathrm{X}_{2}$ & $\mathrm{Z}_{3}$ \\
\hline 100 & $4.75 \mathrm{E}+03$ & 0 & 0 & 0.2353 & 11.176 & -0.0263 & 0.0006 & 1 & 425 \\
\hline 100 & $4.75 \mathrm{E}+03$ & 0 & 0 & 0.7059 & 33.53 & -0.0789 & 0.0017 & 3 & 425 \\
\hline 100 & $4.75 \mathrm{E}+03$ & 0 & 0 & 1.1765 & 55.884 & -0.1315 & 0.0028 & 5 & 425 \\
\hline 100 & $4.75 \mathrm{E}+03$ & 0 & 0 & 1.6471 & 78.239 & -0.1841 & 0.0039 & 7 & 425 \\
\hline 100 & $4.75 \mathrm{E}+03$ & 0 & 0 & 2.1176 & 100.6 & -0.2367 & 0.0050 & 9 & 425 \\
\hline 100 & $4.75 \mathrm{E}+03$ & 0 & 0 & 2.5882 & 122.95 & -0.2893 & 0.0061 & 11 & 425 \\
\hline 100 & $4.75 \mathrm{E}+03$ & 0 & 0 & 3.0588 & 145.32 & -0.3419 & 0.0072 & 13 & 425 \\
\hline 100 & $4.75 \mathrm{E}+03$ & 0 & 0 & 3.5294 & 167.68 & -0.3945 & 0.0083 & 15 & 425 \\
\hline
\end{tabular}

The parallel misalignment Vs Forces and bending stiffness Vs moments are shown in Fig $9 \& 10$.

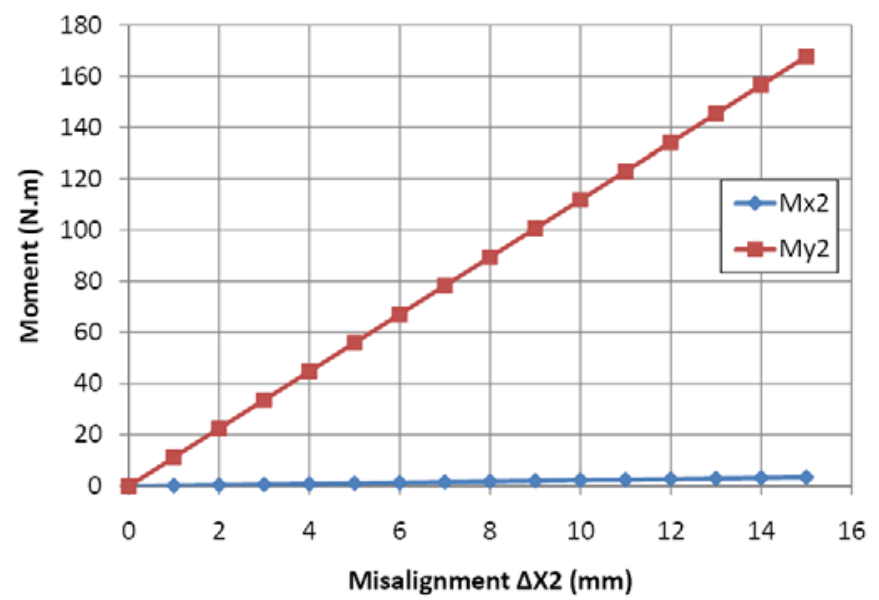

Fig 9. Parallel misalignment Vs end moments

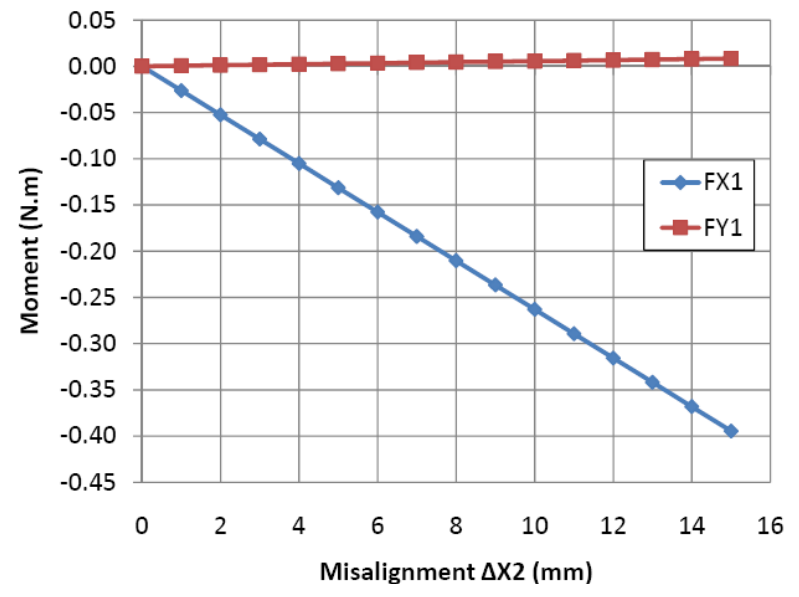

Fig 10. Parallel misalignment Vs Forces

\subsection{Parallel misalignments in different planes Vs Forces and moments}

In this the induced torques, bending stiffness and articulation lengths are kept constant, with the variation in parallel misalignments in different planes Viz. $\Delta \mathrm{X}_{2}$ and $\Delta \mathrm{Y}_{2}$ of the typical HSFC and the resulting forces and moments are found. The results are given in Table 6 .

Table 6 Parallel misalignments in different planes Vs Forces and moments

\begin{tabular}{c|c|c|c|c|c|c|c|c|c|c}
\hline \hline $\begin{array}{c}\text { Torque } \\
(\mathrm{Tq})\end{array}$ & $\mathrm{kb}$ & $\mathrm{Mx} 1$ & $\mathrm{My} 1$ & $\mathrm{Mx} 2$ & $\mathrm{My} 2$ & $\mathrm{FX} 1$ & $\mathrm{FY} 1$ & $\Delta \mathrm{X} 2$ & $\Delta \mathrm{Y} 2$ & $\mathrm{Z} 3$ \\
\hline 100 & $4.75 \mathrm{E}+03$ & 0 & 0 & -10.9412 & 11.412 & -0.0269 & -0.0257 & 1 & 1 & 425 \\
\hline 100 & $4.75 \mathrm{E}+03$ & 0 & 0 & -32.8238 & 34.236 & -0.0806 & -0.0772 & 3 & 3 & 425 \\
\hline 100 & $4.75 \mathrm{E}+03$ & 0 & 0 & -54.7072 & 57.06 & -0.1343 & -0.1287 & 5 & 5 & 425 \\
\hline 100 & $4.75 \mathrm{E}+03$ & 0 & 0 & -76.5918 & 79.886 & -0.1880 & -0.1802 & 7 & 7 & 425 \\
\hline 100 & $4.75 \mathrm{E}+03$ & 0 & 0 & -98.4781 & 102.71 & -0.2417 & -0.2317 & 9 & 9 & 425 \\
\hline 100 & $4.75 \mathrm{E}+03$ & 0 & 0 & -120.3667 & 125.54 & -0.2954 & -0.2832 & 11 & 11 & 425 \\
\hline 100 & $4.75 \mathrm{E}+03$ & 0 & 0 & -142.2580 & 148.38 & -0.3491 & -0.3347 & 13 & 13 & 425 \\
\hline 100 & $4.75 \mathrm{E}+03$ & 0 & 0 & -164.1525 & 171.21 & -0.4029 & -0.3862 & 15 & 15 & 425 \\
\hline
\end{tabular}


With the variation in parallel misalignments in $\Delta \mathrm{X}_{2}$ and $\Delta \mathrm{Y}_{2}$ the increase in moments and reaction forces are observed. The Fig 11 \& 12 shows the parallel misalignments induced in different planes Vs forces and bending moments.

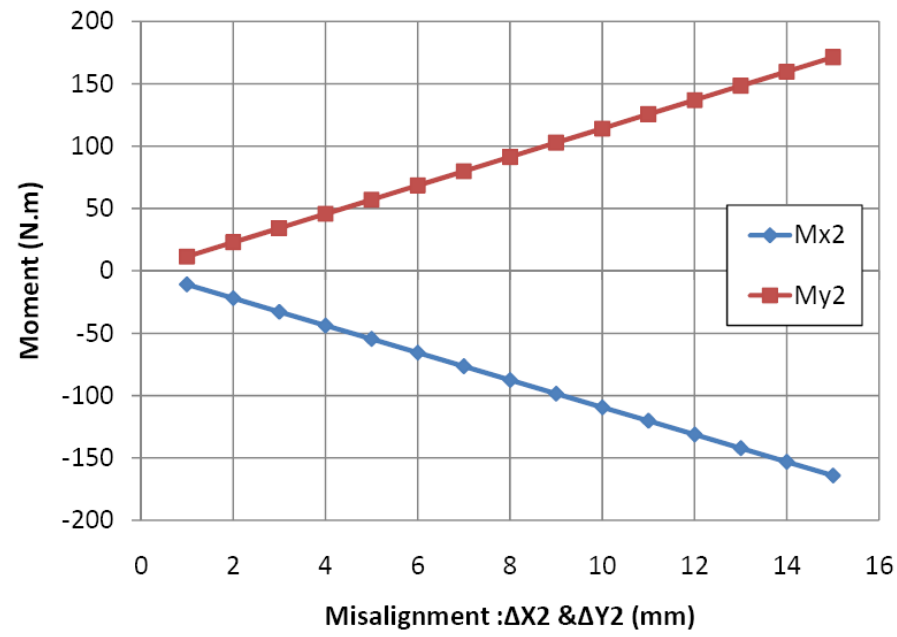

Fig 11. Parallel misalignment in different planes

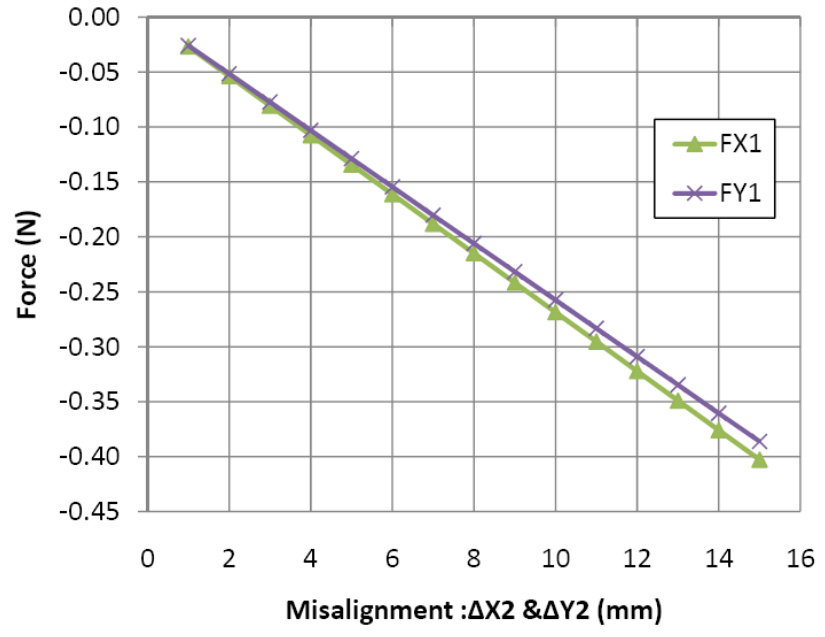

Fig 12. Parallel misalignment in different planes Vs Forces

\section{Vs end moments}

\section{Axial misalignment}

The Axial misalignment of HSFC assembly can be modeled as springs connected in parallel. The HSFC has two flexible plates pack which are acts as springs connected in a parallel manner by centre tube. As the flexible plates pack are in parallel the absolute deflection is equal to the deflection of the system and each flexible plates pack is carrying part of the total load applied such that the total load supported is equal to the sum of the loads carried by individual springs. In present case flexible plates may be considered as spring 1 and spring 2 with axial stiffness of $K_{1}$ and $K_{2}$ respectively as shown in Fig 13. The equivalent axial spring rate is given by eq (16). The axial misalignment (Xa) imposed on the HSFC resulting in the reaction force (F) at the supporting end is given by eq (17).

$$
\begin{aligned}
& \mathrm{K}_{\mathrm{f}}=K_{1}+K_{2} \\
& \mathrm{~F}=\mathrm{K}_{\mathrm{e}} \cdot X_{\sigma}
\end{aligned}
$$

The axial misalignment (imposed on the HSFC and resulting forces (F) at the supporting end are shown in Fig.14.The linear spring rates for the HSFC in axial mode is assumed.

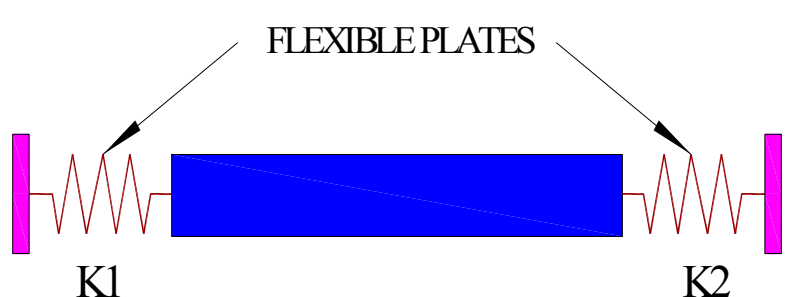

Fig 13. Axial misalignment of HSFC

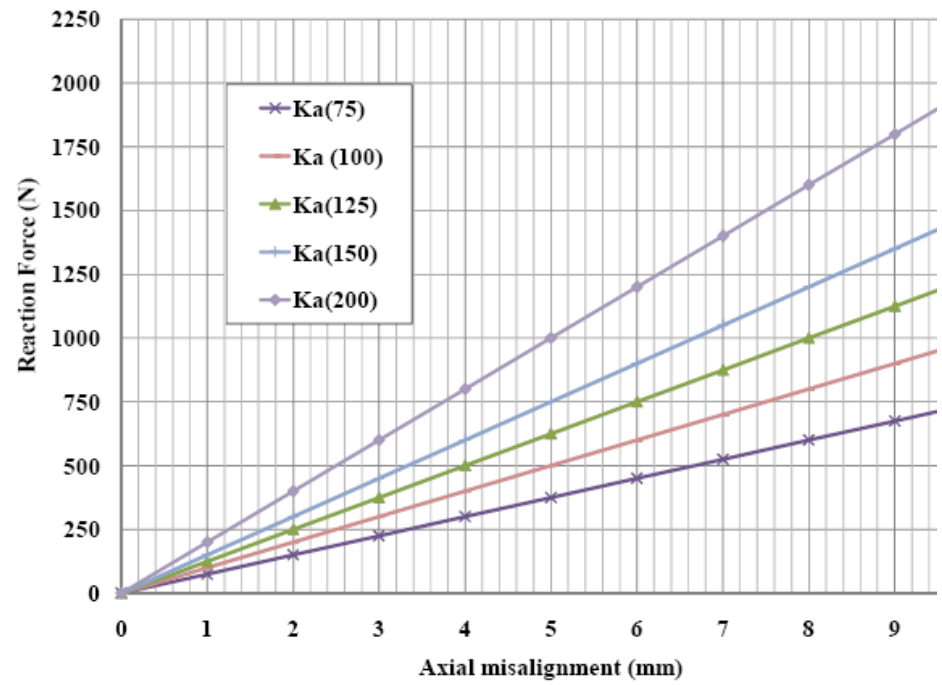

Fig 14. Axial misalignment of HSFC Vs Reaction forces

From the results it is shown that the axial misalignment governed by the axial stiffness of HSFC and lower axial stiffness will be resulting in minimal reaction forces. 


\section{Experimental validation of axial misalignment}

The reaction forces during axial misalignment of the typical HSFC are validated by axial thrust test rig as shown in Fig 15 . The Axial thrust test rig comprises of two mounting flanges for accommodating the test unit. In this, one flange is fixed and other one is movable by the motion imparted by a lead screw and a turning hand wheel arrangement. The extension length is measured in steps of $0.5 \mathrm{~mm}$ by the dial gauge, attached to the structure. The deflection of test unit on extension is measured by the dial gauge and load due to extension is measured by load cell provided on fixed mounting flange. The typical HSFC assembly is subjected to maximum axial misalignment of $2.5 \mathrm{~mm}$ in steps of $0.5 \mathrm{~mm}$ and after completion of the test, the assembly was visually examined and that no evidence of failure and yielding was found. The reaction forces measured are closely agrees with the theoretical predications. The theoretical prediction Vs model behavior of HSFC is shown in Fig 16.

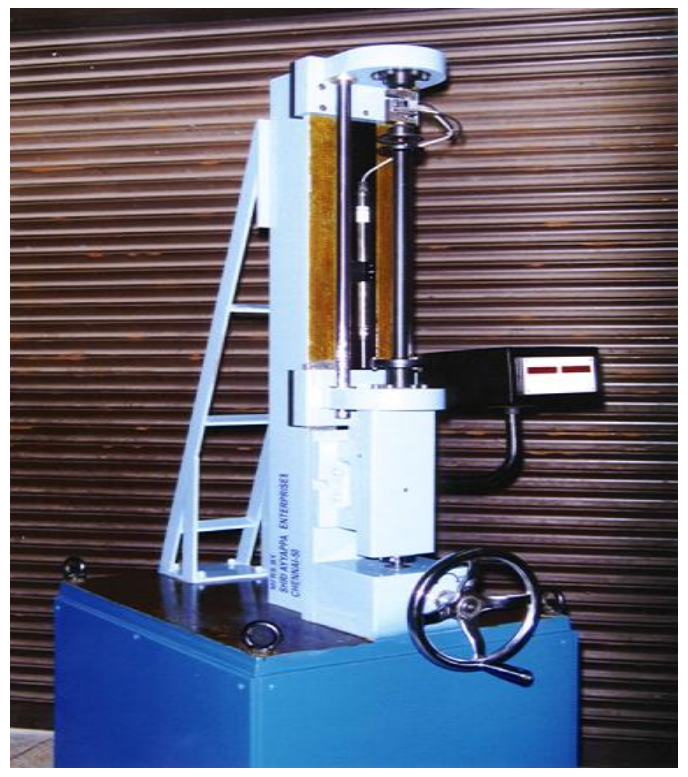

Fig 15. Axial thrust test rig

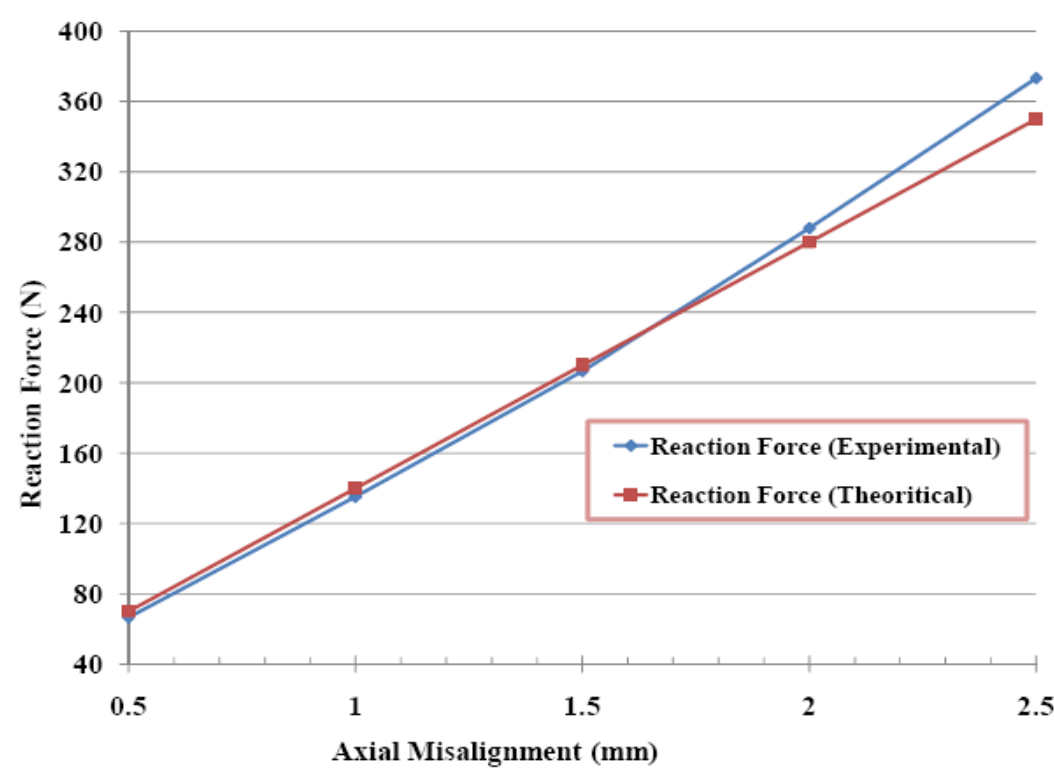

Fig 16.Theoretical prediction Vs Model behavior

\subsection{Combined axial and parallel misalignment test of HSFC}

The typical HSFC developed has been subjected to extensive testing in combined axial and parallel misalignment for 10 million cycles at $12000 \mathrm{rpm}$. The misalignment cycle test rig as shown in Fig 17.The objective of this test is to check and verify the satisfactory endurance performance of typical HSFC. The test rig is configured with a DC motor and a, step up gearbox along with a torque speed sensor. The axial and parallel misalignment of the High speed flexible coupling can be imposed with a special cross table screw arrangement at the drive end.

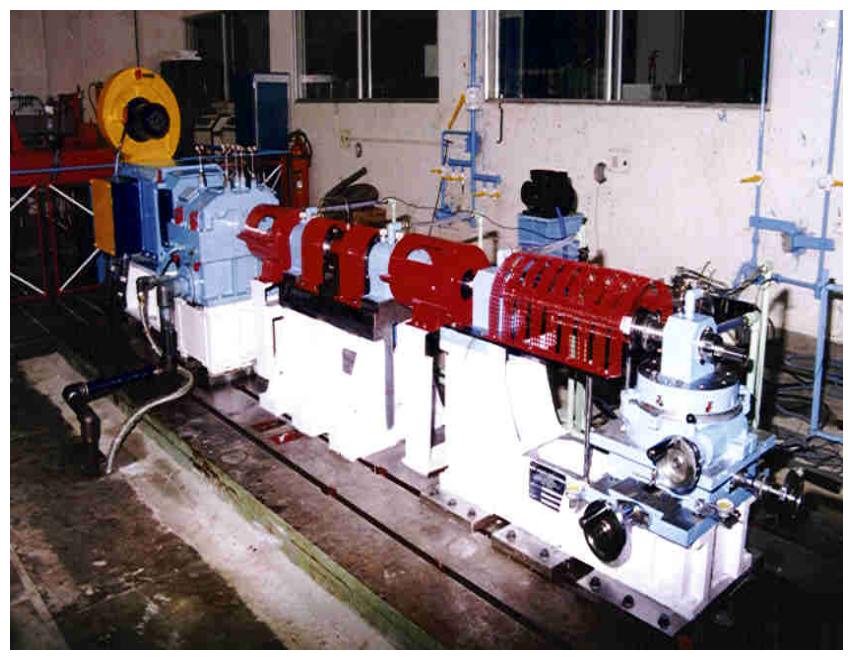

Table 7 Combined axial and parallel misalignment test conditions of HSFC

\begin{tabular}{c|c|c|c|c}
\hline \hline Sl. No & Speed & $\begin{array}{c}\text { Parallel } \\
\text { misalignment } \\
(\mathrm{mm})\end{array}$ & $\begin{array}{c}\text { Axial } \\
\text { misalignment } \\
(\mathrm{mm})\end{array}$ & Cycles \\
(revolutions)
\end{tabular}

Fig 17. Misalignment cycle test rig

The HSFC assembly was mounted and drive end flange is imposed with equivalent parallel and axial misalignment. During testing, speed, vibrations at the input and the output ends and noise level are monitored and recorded. The axial misalignment up to $2.5 \mathrm{~mm}$ and parallel misalignment up to $1.85 \mathrm{~mm}$ was imposed. For each load step, the testing was conducted for 10 million cycles. After completion of the test, the HSFC assembly was visually examined and found no evidence of failure and yielding which shows that the bending moments and reaction forces are well within the acceptable limits. 


\section{Discussion}

The effect of various misalignment of HSFC on the Fighter aircraft transmission characteristics has been analyzed. The articulation length of the HSFC influences the parallel misalignment accommodating capability of HSFC. The analysis performed clearly shows that as the articulation length of the coupling increases the corresponding moment and forces on the end support are decreasing. Hence it is advantageous to place the flexible plate packs as closer to the support ends as the corresponding bending stress induced on the flexible elements will be less thereby increase in the HSFC service life. The HSFC's bending stiffness to be designed as lowest as possible. The increase in parallel misalignment in multiple planes will result in increase in support end forces and bending moments. This has to be carefully studied for the rating of HSFC parallel misalignment capability .During installation care to be taken to align the HSFC to reduce the static misalignment. The axial misalignment governed by the axial stiffness of HSFC and with lower axial stiffness the corresponding reaction forces will be minimum. From the experimental evaluation of forces due to axial misalignment, minor variation in theoretical prediction Vs model behavior is observed. This is may be due to the friction present in the test set up. The successful performance of HSFC in combined axial and parallel misalignment test indicates that the bending moments and reaction forces are well within the acceptable limits. The suitability of the model adapted for the design iteration of HSFC has been successfully implemented.

\section{Conclusion}

In the present study, influence of articulation length and effect of reaction force and moments experienced by the coupling has been carried out. The coupling misalignment forces and moments have been determined for both parallel and angular misalignments of a HSFC. It is observed that the coupling can be designed to accommodate more parallel misalignment by positioning the flexible elements with optimized articulation length. The axial misalignment capability of the shaft is sole function of axial stiffness of the flexible elements. This observation will be useful in designing of compact flexible coupling where higher misalignment capability within the available space has to be met. With the experimentation, the suitability of the model adapted for the design iteration of HSFC development is validated. This method will reduce the design iteration cycles of HSFC and can be extended for the similar development of flexible couplings.

\section{Nomenclature}

\begin{tabular}{|c|c|c|c|}
\hline $\mathrm{FX}_{1}$ & forces in $\mathrm{X} 1$ direction, $[\mathrm{N}]$ & $\mathrm{Tq}$ & torque, $[\mathrm{N}-\mathrm{mm}]$ \\
\hline $\mathrm{FX}_{2}$ & forces in $\mathrm{X} 2$ direction, $[\mathrm{N}]$ & $\mathrm{Xa}$ & axial misalignment, $[\mathrm{mm}]$ \\
\hline $\mathrm{FY}_{1}$ & forces in $\mathrm{Y} 1$ direction, $[\mathrm{N}]$ & $\mathrm{Z}_{3}$ & articulation length, $[\mathrm{mm}]$ \\
\hline $\mathrm{FY}_{2}$ & forces in $\mathrm{Y} 2$ direction, $[\mathrm{N}]$ & $\theta_{1}$ & Rotating angle in $\mathrm{X}_{1}$ direction, $[\mathrm{deg}]$ \\
\hline $\mathrm{K}_{\mathrm{a}}$ & axial stiffness, $[\mathrm{N} / \mathrm{mm}]$ & $\theta_{2}$ & Rotating angle in $\mathrm{X}_{2}$ direction, $[\mathrm{deg}]$ \\
\hline $\mathrm{Kb}$ & bending stiffness, [N.mm/deg] & $\theta_{3}$ & misalignment angle, $[\mathrm{deg}]$ \\
\hline $\mathrm{Ke}$ & $\begin{array}{l}\text { equivalent axial stiffness of flexible } \\
\text { plate packs, }[\mathrm{N} / \mathrm{mm}]\end{array}$ & $\Phi_{1}$ & Rotating angle in $\mathrm{Y}_{1}$ direction, $[\mathrm{deg}]$ \\
\hline $\mathrm{K}_{1}, \mathrm{~K}_{2}$ & $\begin{array}{l}\text { axial stiffness of Flexible plate packs, } \\
{[\mathrm{N} / \mathrm{mm}]}\end{array}$ & $\Phi_{2}$ & Rotating angle in $\mathrm{Y}_{2}$ direction, $[\mathrm{deg}]$ \\
\hline $\mathrm{MX}_{1}$ & moments in $\mathrm{X}_{1}$ direction, [N-mm] & $\Delta \mathrm{X}_{1}$ & misalignment displacement on $\mathrm{X}_{1}$ direction, $[\mathrm{mm}]$ \\
\hline $\mathrm{MX}_{2}$ & moments in $\mathrm{X}_{2}$ direction, $[\mathrm{N}-\mathrm{mm}]$ & $\Delta \mathrm{X}_{2}$ & misalignment displacement on $\mathrm{X}_{2}$ direction, $[\mathrm{mm}]$ \\
\hline $\mathrm{MY}_{1}$ & moments in $\mathrm{Y}_{1}$ direction, $[\mathrm{N}-\mathrm{mm}]$ & $\Delta \mathrm{Y}_{1}$ & misalignment displacement on $\mathrm{Y}_{1}$ direction, $[\mathrm{mm}]$ \\
\hline $\mathrm{MY}_{2}$ & moments in $\mathrm{Y}_{2}$ direction, $[\mathrm{N}-\mathrm{mm}]$ & $\Delta \mathrm{Y}_{2}$ & misalignment displacement on $\mathrm{Y}_{2}$ direction, $[\mathrm{mm}]$ \\
\hline
\end{tabular}

\section{References}

[1] Nagesh.S., Ganesan.S. and Chandra sekaran C., 2004, "Design and Development of Power take off shaft with contoured diaphragms for Aircraft applications DRDO work shop on advanced manufacturing," DRDL, Hyderabad, pp. 784-794.

[2] ANSI/AGMA 9009-D02: "Flexible Couplings- Nomenclature for Flexible Couplings".

[3] Agnieszka Muszynska, 2005, "Rotor dynamics," CRC Press Taylor \& Francis Group.

[4] C.B.Gibbons, 1976, "Coupling misalignment forces," Proceedings of the Fifth Turbo machinery Symposium Gas Turbine Laboratories $\backslash$ Texas A \& M University, College Station, Texas, pp. 111-116.

[5] A.S. Sekhar and B.S. Prabhu, 1995, "Effects of coupling misalignment on vibrations of rotating machinery," Journal of Sound and Vibration, Vol. 185 (4), pp. 655-671.

[6] Nagesh S., 2000, "Lateral critical speed analysis of high speed shaft with contoured diaphragms using transfer matrix Method," M.E. thesis, PSG college of Technology, Coimbatore. 